\title{
Parametric imaging of viscoelasticity using optical coherence elastography
}

\author{
Philip Wijesinghe ${ }^{1, *}$, Robert A. McLaughlin ${ }^{1}$, David D. Sampson ${ }^{1,2}$ and Brendan \\ F. Kennedy ${ }^{1}$ \\ ${ }^{1}$ Optical+Biomedical Engineering Laboratory, School of Electrical, Electronic \& \\ Computer Engineering, The University of Western Australia, Crawley, Australia \\ ${ }^{2}$ Centre for Microscopy, Characterisation \& Analysis, The University of Western \\ Australia, Crawley, Australia \\ *Email: philip.wijesinghe@research.uwa.edu.au
}

\begin{abstract}
We demonstrate imaging of soft tissue viscoelasticity using optical coherence elastography. Viscoelastic creep deformation is induced in tissue using step-like compressive loading and the resulting time-varying deformation is measured using phase-sensitive optical coherence tomography. From a series of co-located B-scans, we estimate the local strain rate as a function of time, and parameterize it using a four-parameter Kelvin-Voigt model of viscoelastic creep. The estimated viscoelastic strain and time constant are used to visualize viscoelastic creep in two-dimensional, dual-parameter viscoelastograms. We demonstrate our technique on six silicone tissue-simulating phantoms spanning a range of viscoelastic parameters. As an example in soft tissue, we report viscoelastic contrast between muscle and connective tissue in fresh, ex vivo rat gastrocnemius muscle and mouse abdominal transection. Imaging viscoelastic creep deformation has the potential to provide complementary contrast to existing imaging modalities, and may provide greater insight into disease pathology.
\end{abstract}

\section{Introduction}

Disease commonly alters tissue mechanical properties (Friedl and Alexander 2011; Ingber 2003; Mierke 2012; Papandrinopoulou et al. 2012; Yoon et al. 2013). The measurement of disease-altered mechanical properties can serve as a tool for identification and characterization of disease state and progression (Discher et al. 2009; Geyer et al. 2004; Lee and Lim 2007; Parker et al. 2011; Qin et al. 2010). Over the past 25 years, a family of imaging techniques, known as elastography, have been developed to image 
the mechanical properties of tissue (Parker et al. 2011). Each technique consists of three key steps: (1) a mechanical load is imparted to a tissue; (2) the resulting deformation is measured using an imaging technique; and (3) a mechanical property is estimated and mapped into an image, known as an elastogram. Elastography, based on either ultrasound or magnetic resonance imaging (MRI), has matured to the point of commercial availability. Examples of clinical applications of elastography include liver fibrosis (Sandrin et al. 2003) and breast cancer (Chang et al. 2011).

Amongst various optical approaches to elastography, a number of optical coherence tomography (OCT)-based techniques, known collectively as optical coherence elastography (OCE), have been proposed. These techniques have the potential to improve the spatial resolution and sensitivity to deformation over ultrasound and MRI-based techniques. OCE has been proposed and to some degree demonstrated for a range of applications, including in breast cancer (Srivastava et al. 2011; Liang et al. 2008; Kennedy et al. 2014c), ophthalmology (Alonso-Caneiro et al. 2011; Ford et al. 2011; Wang and Larin 2014), and cardiology (Chan et al. 2004; Chau et al. 2004; Qi et al. 2012). OCE techniques may be conveniently classified by the mechanisms used to impart the mechanical load, and by the methods and models used to extract mechanical properties from the measured deformation (Kennedy et al. 2014a). Examples include: compression (Kennedy et al. 2009; Nahas et al. 2013), surface acoustic wave (SAW) and shear wave (SW) (Song et al. 2013), and magnetomotive (MM) (Oldenburg and Boppart 2010) OCE. The majority of OCE techniques have probed the mechanical properties of tissue under the assumption that tissue is purely elastic (Kennedy et al. 2014a), i.e., that the tissue's mechanical response is adequately modelled by a network of connected springs. However, tissue exhibits viscoelasticity, combining characteristics of both solids and liquids. Viscoelasticity is more accurately modelled as a system of springs and dampers (Tschoegl 1989). The appropriate parameterization of the viscoelastic properties of soft tissue has the potential to provide contrast that is complementary to purely elastic contrast and to provide a more complete characterization of tissue mechanics. For example, in the related field of ultrasound elastography, characterization of viscoelasticity has shown the capacity to differentiate malignant from benign breast cancer tissues (Qiu et al. 2008), and has shown promise in characterizing rodent mammary tumours (Wang and Insana 2013).

Prior work using optical techniques has demonstrated the potential of viscoelasticity in point-based measurements. In one study on agarose gel, viscoelastic creep in response to an indentation load was measured using OCT (Yang et al. 2007). Using a spherical indentation model, point measurements of viscosity were derived from the displacement of material interfaces observed in OCT B-scan images. In another technique, viscoelastic creep was measured using MM-OCE through step-like excitation of magnetic nanoparticles embedded in tissue (Crecea et al. 2013). Tissue viscoelasticity was characterized using motion- (M-) mode, phase-sensitive OCE, where axial displacement is derived from the phase difference of successive co-located axial scans (Wang et al. 2007). In a related technique, referred to as creep compounding, the viscoelastic response of tissue was used to reduce speckle in OCT (Kennedy et al. 2011).

In this paper, we demonstrate the capacity of OCE to perform parametric imaging of tissue viscoelasticity on sub-millimetre resolution scales. Using phase-sensitive OCT, we measure the time- 
dependent response of a sample subjected to a step load. Using processing techniques similar to those previously developed for compression OCE (Kennedy et al. 2012), we measure the strain rate between two OCT B-scans. By fitting the strain rate versus time to a model of viscoelastic creep deformation, we extract both the viscoelastic strain and time constant. In an extension of previous techniques, we present B-scan images of viscoelasticity (viscoelastograms) using a two-parameter (viscoelastic strain and time constant) colour scale that distinguishes sample regions with distinct viscoelastic properties. We demonstrate our technique on tissue-mimicking phantoms with controlled viscoelastic properties. We present dual-parameter viscoelastograms of freshly excised rat gastrocnemius muscle and mouse abdominal muscle that demonstrate contrasting features. Parametric imaging of viscoelasticity with OCE may provide opportunities for characterizing tissue and disease mechanics on the sub-millimetre scale, and provide new diagnostic tools complementary to other OCE methods.

\section{Methods}

\subsection{System set-up and loading protocol}

Imaging was performed using a fibre-based spectral-domain OCT system, previously described in detail by Kennedy et al. (2014c). A superluminescent-diode source was used, with a central wavelength of 835 $\mathrm{nm}$ and a bandwidth of $50 \mathrm{~nm}$. To improve phase-sensitive detection, the system was set up in a common-path configuration, in which the reflection from the sample side of the imaging window (IW, figure 1) was used as the reference signal. The imaging window consists of a 2-mm thick glass plate, which acted to support the sample and provide reaction forces to applied loads. The measured axial and lateral resolutions are $8.5 \mu \mathrm{m}$ (in air) and $11 \mu \mathrm{m}$, respectively. Images were acquired with a sample-arm illumination power of between 7 and $8 \mathrm{~mW}$. In this paper, axial distances are given as optical lengths, i.e., product of the group refractive index and physical length, and lateral distances are given as physical lengths.

To induce viscoelastic creep deformation (Tschoegl 1989), a flanged cylindrical weight (30 mm height and $25 \mathrm{~mm}$ diameter body, with an additional $5 \mathrm{~mm}$ height and $30 \mathrm{~mm}$ diameter head) providing a constant gravitational load (GL) of $1 \mathrm{~N}$ was placed on the sample, as shown in figure 1 . The load was constrained in all its degrees of freedom, except axially, by a ring holder (RH) which guided the load without being connected to it. The ring holder was mounted on an actuated (z-) translation stage (TS), and was manually aligned to ensure that the contact surface of the gravitational load was coaxial with the optical axis and coplanar with the imaging window. The actuated stage was used to guide the gravitational load thereby providing controllable and repeatable placement of the load onto the sample. Prior to acquisition, the stage was manually lowered until the first point of contact of the gravitational load with the sample surface, such that minimal preload deformation was introduced prior to imaging. The sample was allowed to relax until no displacements above the noise floor of the system (described in Section 2.3) were observed. The stage was then lowered at a rate of $0.2 \mathrm{~mm} / \mathrm{s}$ for $4 \mathrm{~s}$, synchronized with the start of acquisition. Imaging was performed from the opposite side of the sample to that being loaded. 

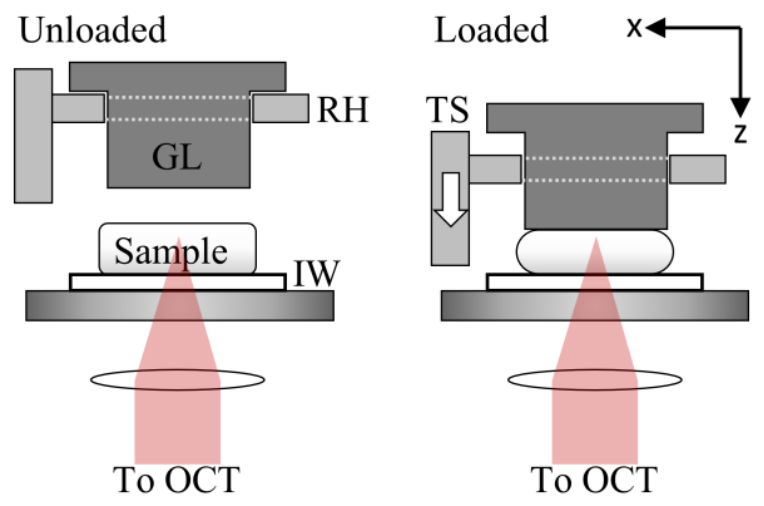

Figure 1. Schematic of the sample arm loading system, with the sample unloaded (left) and loaded (right). GL, gravitational load; RH, ring holder; TS, translation stage; and IW, imaging window.

\subsection{Viscoelastic model}

Under constant load, a viscoelastic material deforms over time at a decreasing rate until a steady state is reached. This behaviour is termed viscoelastic creep deformation (Tschoegl 1989). It can be observed on the macro-scale, as whole body deformation, as well as on the micro-scale, where collections of cells, tissue constituents and other micro-structures deform under the bulk body loading.

To model viscoelastic creep deformation within tissue, we employ a four-parameter Kelvin-Voigt model, consisting of a parallel spring and damper unit with a series of spring and damper elements (Tschoegl 1989), as illustrated in the inset in figure 2. In this model, the time-dependent strain, $\varepsilon(t)$, shown in figure 2(a), is described by the following equation:

$$
\varepsilon(t)=\varepsilon_{0}+\varepsilon_{1}\left[1-\exp \left(-\frac{t}{\tau}\right)\right]+\varepsilon_{2} t,
$$

where $\varepsilon_{0}$ represents the instantaneous 'elastic' strain; $\varepsilon_{1}$ and $\tau$ represent the 'viscoelastic' strain and time constant, respectively; and $\varepsilon_{2}$ represents the 'viscous' strain (Sridhar and Insana 2007). The contribution of these parameters to the viscoelastic creep deformation is illustrated in figure 2 . The contribution of the viscous element, $\varepsilon_{2}$, does not diminish with time and represents a steady-state loss of volume. It is typically attributed to the viscous flow of free liquid present within a material (Sridhar and Insana 2007). For example, in tissue this can account for the exudation of blood, lymph, or intraand extra-cellular fluids.

Such a characterization of viscoelastic creep deformation is in part quantitative and in part a relative measure. The three strain terms in equation (1) represent relative measures of deformation. A value of stress, $\sigma$, is required to convert them to a mechanical modulus, such as creep modulus, $G_{c}=\sigma / \varepsilon_{1}$. By contrast, $\tau$ is directly quantifiable and independent of the applied stress (Tschoegl 1989). 

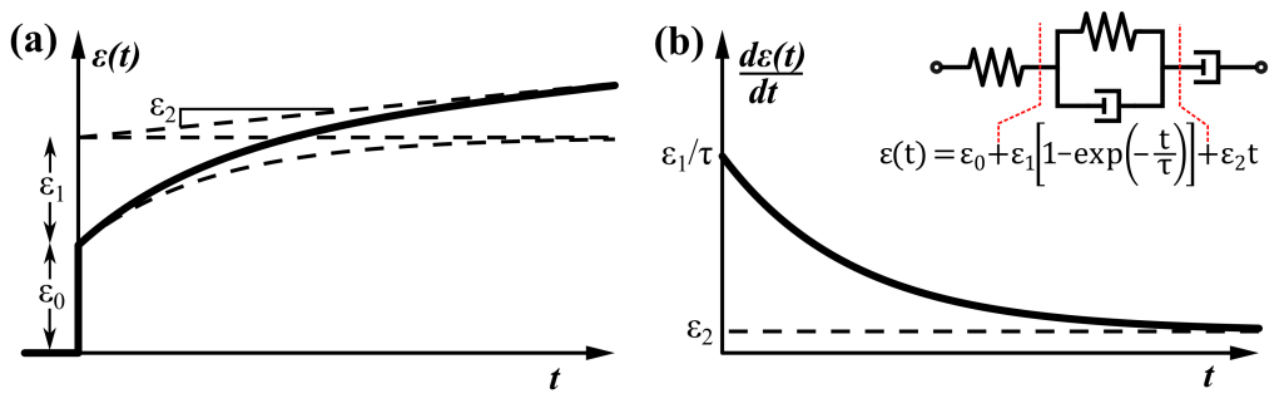

Figure 2. (a) Viscoelastic creep strain and (b) viscoelastic creep strain-rate response of a fourparameter Kelvin-Voigt model to a constant load. Inset shows the four-parameter Kelvin-Voigt model represented as a spring and damper system.

\subsection{Data analysis and acquisition}

We parameterize the viscoelastic response within tissue using two of the four model parameters, the viscoelastic strain, $\varepsilon_{1}$, and viscoelastic time constant, $\tau$. To achieve this, we adapt a method used in phase-sensitive compression OCE (Kennedy et al. 2012) to estimate the change in local strain from sample displacement measured between pairs of co-located, complex OCT B-scans. We extract the local sample displacements from the phase difference between the B-scans (Wang et al. 2007). Local strain, $\varepsilon(t)$, is defined as the spatial derivative of displacement averaged over a finite axial depth range within a sample (Kennedy et al. 2012). The change in local strain, $\Delta \varepsilon(t)$, is defined as the deformation measured between two B-scans, i.e., $\Delta \varepsilon(t)=\varepsilon(t+\Delta t)-\varepsilon(t)$, where $\Delta t$ is the time between the B-scans. From the local, axial variations in the measured displacements, the changes in local strain are estimated using a weighted-least squares (WLS) approach (Kennedy et al. 2012), with an axial fitting range of 50 $\mu \mathrm{m}$.

A limitation of phase-sensitive OCE is that displacements exceeding one half of a wavelength are ambiguous due to phase wrapping. Characterizing the viscoelastic response is particularly susceptible to this limitation because of the relatively large displacements in the early response. Therefore, without phase unwrapping, we cannot estimate local strain, $\varepsilon(t)$, necessary for parameterization by equation (1), as it requires the integration of strain rate at every time point, which is confounded by the phase-wrapped region. By differentiating equation (1), we instead derive the strain rate representation of the model, as illustrated in figure $2(b)$, i.e.,

$$
\frac{\Delta \varepsilon(t)}{\Delta t} \approx \frac{\mathrm{d} \varepsilon(t)}{\mathrm{d} t}=\frac{\varepsilon_{1}}{\tau} \exp \left(-\frac{t}{\tau}\right)+\varepsilon_{2}
$$

For simplicity, the strain rate data corrupted by the presence of phase wrapping is discarded; therefore, the remaining data can be fitted directly by equation (2), providing an estimate of the viscoelastic parameters $\varepsilon_{1}$ and $\tau$. For long acquisition times, where $t \gg \tau$, a steady state is reached, where the exponential decay in strain rate approaches zero and the strain rate model becomes dominated by $\varepsilon_{2}$. 
Averaging the measured strain rate observed at the end of the response results in an estimate of $\varepsilon_{2}$. This reduces the fit parameters from 3 to 2, improving the robustness of fit for $\varepsilon_{1}$ and $\tau$, and increases the data processing speed.

Viscoelastic parameters $\varepsilon_{1}$ and $\tau$ were estimated by fitting equation (2), including the estimate of $\varepsilon_{2}$, to the measured strain rate by minimizing the weighted sum-of-squares of residuals. The measured strain rate was also spatially filtered using a rectangular window (boxcar) smoothing kernel prior to fitting to reduce the effect of noise. Below, window size is specified for all presented results.

We acquired B-scans at a rate of $10 \mathrm{~Hz}$. Changes in local strain were estimated for each consecutive acquisition pair, resulting in $\Delta \varepsilon(t)$ measurements every $\Delta t=0.1 \mathrm{~s}$. For each B-scan, $1000 \mathrm{~A}$-scans were acquired over a $10 \mathrm{~mm}$ field-of-view at rate of $10 \mathrm{kHz}$, with the exception of the experiment on the duallayer tissue-simulating phantom (described in Section 2.4), where $500 \mathrm{~A}$-scans over a $5 \mathrm{~mm}$ field-ofview were acquired at a rate of $5 \mathrm{kHz}$. For all experiments, B-scan acquisition commenced immediately prior to load application. Total acquisition time was approximately $40 \mathrm{~s}$ for the stiff phantoms, $80 \mathrm{~s}$ for soft phantoms, and $120 \mathrm{~s}$ for tissue. OCT acquisition times were chosen, by observation, to encompass the full viscoelastic creep response, described in Section 2.2, from loading to steady-state. The displacement and strain sensitivity of the phase-sensitive OCE system was measured to be $0.34 \mathrm{~nm}$ and $2.6 \mu \varepsilon(100 \mu \mathrm{m}$ axial fitting range), respectively (Kennedy et al. 2014c).

\subsection{Sample Preparation}

The viscoelastic imaging technique described here was initially evaluated using tissue-simulating phantoms fabricated from room-temperature vulcanizing (RTV) silicone rubber (Elastosil® P7676 and Elastosil ${ }^{\circledR}$ RT601, Wacker, Germany) and polydimethylsiloxane (PDMS) oil (AK50, Wacker, Germany). Titanium dioxide particles were added to introduce optical scattering. The fabrication procedure is described in detail by Lamouche et al. (2012). Phantoms were cylindrical in shape, with a height of $3 \mathrm{~mm}$ and a diameter of $22 \mathrm{~mm}$. Variation in mechanical properties was achieved by using different mixing ratios of silicone compound (Part A), cross-linker (Part B) and PDMS oil. The composition of the silicone phantoms is specified by the relative proportions of these components, e.g., RT601 1:10:10. Additional PDMS oil was used as a lubricant on the imaging plate and compression surface to reduce friction. The oil also acted as a refractive index-matching fluid.

Imaging was performed on a series of homogeneous silicone phantoms of different composition, and a dual-layer phantom. Homogeneous phantom compositions were selected to encompass a range of elastic moduli. These compositions are described in detail elsewhere (Lamouche et al. 2012). The elastic modulus (at $1 \mathrm{~N}$ load) of the phantoms is listed in table 1 . These values were measured by uniaxial compression using an Instron 5848 micro tester system (Instron, Norwood, MA, USA). Homogeneous phantoms were fabricated with optical scatterer density of $1 \mathrm{mg} / \mathrm{mL}$. The dual-layer phantom was constructed from two silicone compositions with contrasting mechanical properties: a $2.5-\mathrm{mm}$ thick layer of P7676 2:1:0.3 and a thin 0.4-mm thick layer of RT601 5:1:0 with matching scatterer density of $1 \mathrm{mg} / \mathrm{mL}$. The dual-layer phantom was orientated with the thin layer towards the imaging side, as shown 
in figure 3(a). As described previously (de Bruin et al. 2010), the quantity of titanium dioxide particles added results in a scattering coefficient within the range expected in tissue.

Viscoelastic imaging was also performed on freshly excised rat and mouse muscle tissue samples, obtained under The University of Western Australia Animal Ethics Committee's tissue sharing protocols. For rat gastrocnemius muscle, the OCT beam was incident upon the dorsal side of the muscle with the B-scan aligned transverse to the muscle fibres. For mouse abdominal muscle, the sample was imaged from the peritoneal side (organ side) with B-scan cross-section along the sagittal plane (vertical plane). We placed the rat and mouse muscle on top of a thin $(500 \mu \mathrm{m})$ compliant silicone layer, fabricated from P7676 2:1:0.3 with no added scatterers, to distribute the applied compressive load over the irregular surface geometry of tissue (Kennedy et al. 2014d). Orientation of animal tissue over the compliant layer is shown schematically in figure 3(b). After excision, the tissue samples were kept hydrated until imaging $(<5$ hours) in saline solution, which was also used as lubrication for both the imaging and compression surfaces.

(a)

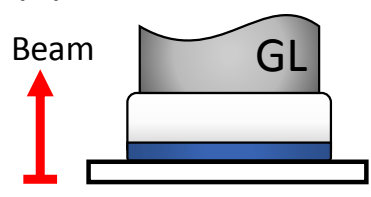

(b)

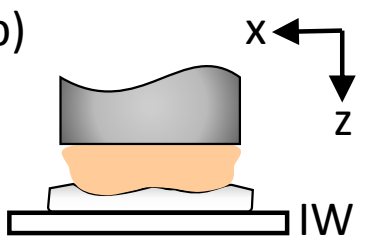

Figure 3. Sample positioning in the sample arm loading system, showing (a) dual-layer phantom (thick layer, white; thin layer, blue), and (b) animal tissue (light brown) on top of a compliant silicone layer (white); compressed between the gravitational load (GL) and the imaging window (IW); OCT incident beam direction shown with a red arrow.

\section{Results}

\subsection{Homogeneous phantoms}

Three independent OCT acquisitions were performed on six tissue-simulating phantoms. For each acquisition, the measured strain rate was spatially averaged across a $5-\mathrm{mm}$ lateral and $100-\mu \mathrm{m}$ axial range. Measurements were laterally centred in the $10-\mathrm{mm}$ field of view, with the axial range starting at $200 \mu \mathrm{m}$ in depth to minimize boundary friction effects but still ensure a high signal-to-noise ratio of the viscoelastic response. The strain rate response of each acquisition is presented in figure 4(a).

From the measured strain rate curves, we can see that each phantom composition yields a distinct and repeatable viscoelastic response. The calculated values of $\varepsilon_{1}$ and $\tau$ for each acquisition are presented in the parameter map shown in figure 4(b). The bounding ellipses were centred at parameter means and extend to the $95 \%$ confidence intervals of the curve fit. The wide coverage of the parameter space demonstrates the ability of the proposed imaging method to provide versatile viscoelastic contrast. The tight grouping of parameters for each phantom composition suggests that the viscoelasticity of the 
imaged silicone phantoms does not vary significantly through repetitive loading (minimal plastic deformation or long relaxation times), and demonstrates the repeatability of our technique.

The mean, $\mu$, and coefficient of variation, $\sigma / \mu$, of the estimated viscoelastic parameters are presented in table 1 . From the coefficient of variation, we can see that the sensitivity of the imaging system to parameters $\varepsilon_{1}$ and $\tau$ reduces for phantoms with relatively large (e.g., P7676 4:1:0) or small (e.g., RT601 $5: 1: 0)$ viscoelastic parameters, and approximately spans the system's dynamic range. We can adjust this dynamic range by varying imaging parameters, such as loading force and B-scan acquisition speed, bringing different ranges of the viscoelastic response into the observable window, which is further discussed in Section 4.
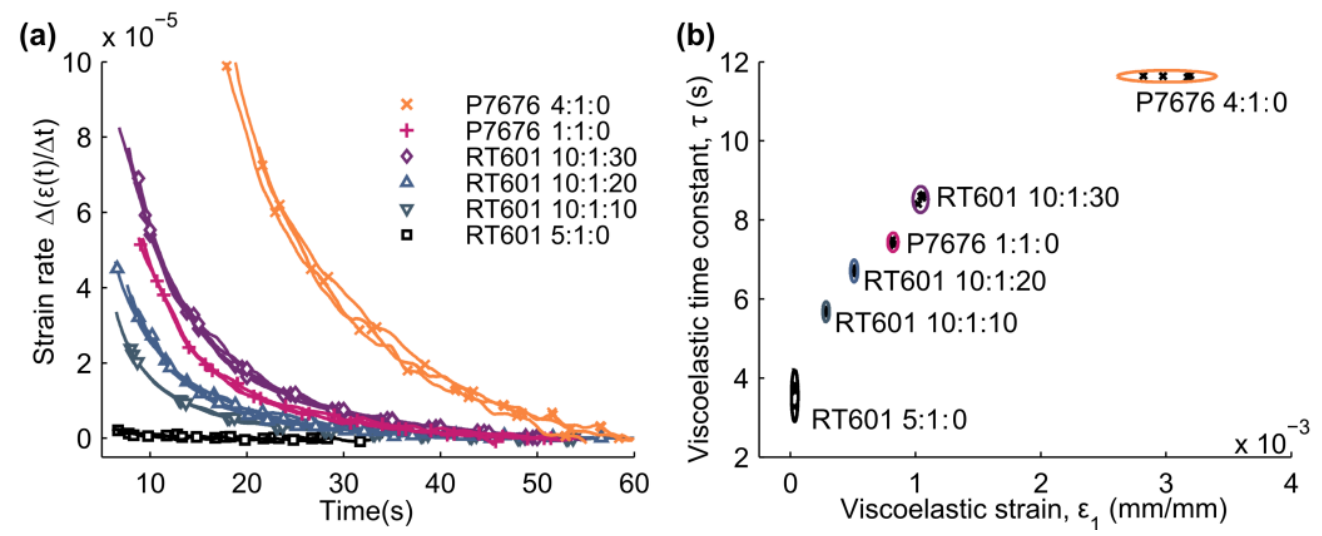

Figure 4. (a) Measured viscoelastic creep strain rate from three independent acquisitions performed on silicone phantoms of different compositions. (b) Viscoelastic strain and time constant parameters estimated from responses in (a), where the ellipses represent the $95 \%$ confidence intervals.

Table 1. Elastic modulus (E) (Instron) and estimated viscoelastic parameters of homogeneous silicone tissue-simulating phantoms

\begin{tabular}{lcccccc}
\hline & $\mathrm{E}$ & \multicolumn{2}{c}{$\varepsilon_{1}$} & & \multicolumn{2}{c}{$\tau$} \\
\cline { 3 - 4 } \cline { 6 - 7 } Material & $(\mathrm{kPa})$ & Mean $\left(\times 10^{-3}\right)$ & $\sigma / \mu(\%)$ & & Mean $(\mathrm{s})$ & $\sigma / \mu(\%)$ \\
\hline P7676 4:1:0 & 3.5 & 3.0 & 5.8 & & 11.6 & 0.2 \\
P7676 1:1:0 & 35 & 0.82 & 1.3 & & 7.4 & 1.0 \\
RT601 10:1:30 & 42 & 1.0 & 1.7 & & 8.6 & 1.2 \\
RT601 10:1:20 & 100 & 0.51 & 0.8 & & 6.7 & 1.1 \\
RT601 10:1:10 & 210 & 0.29 & 1.0 & & 5.6 & 1.2 \\
RT601 5:1:0 & 130 & 0.037 & 8.6 & & 3.6 & 6.7 \\
\hline
\end{tabular}

\subsection{Dual-layer tissue-simulating phantom}

Imaging of viscoelasticity using OCE was performed on a dual-layer phantom. As described in Section 2.4, the phantom layers had matching optical properties and differing mechanical properties. The OCT image is presented in figure 5(a). The layer boundary was measured to be at an optical depth of $570 \mu \mathrm{m}$ ( $410 \mu \mathrm{m}$ physical depth, assuming PDMS refractive index $\approx 1.4$ (Lamouche et al. 2012)) and is indicated 
by the dashed white line. We fitted the viscoelastic parameters to the estimated local strain rates after spatially averaging with a $250-\mu \mathrm{m}$ lateral and $70-\mu \mathrm{m}$ axial rectangular window. Viscoelastic strain, $\varepsilon_{1}$, and time constant, $\tau$, were overlaid on the OCT image to provide a graphical representation of the viscoelastic response, and are presented in figures 5(b) and 5(c), respectively. We observe good contrast between the layers present in both of the parameter maps. The measured $\varepsilon_{1}$ and $\tau$ values in the stiffer (top) layer were $9 \pm 1 \times 10^{-5}$ and $4.5 \pm 0.9 \mathrm{~s}$ (mean \pm standard deviation), respectively, and $1.4 \pm 0.3 \times 10^{-3}$ and $11 \pm 1 \mathrm{~s}$ for the softer (bottom) layer.

We may represent viscoelasticity as a variation in solely $\varepsilon_{1}$ (figure 5(b)), variation in solely $\tau$ (figure 5(c)), or as a combination of both (figure 5(d)), where in each case the parameter is overlaid on the OCT image. The dual-parameter viscoelastogram presented in figure 5(d) exhibits strong contrast between the layers.
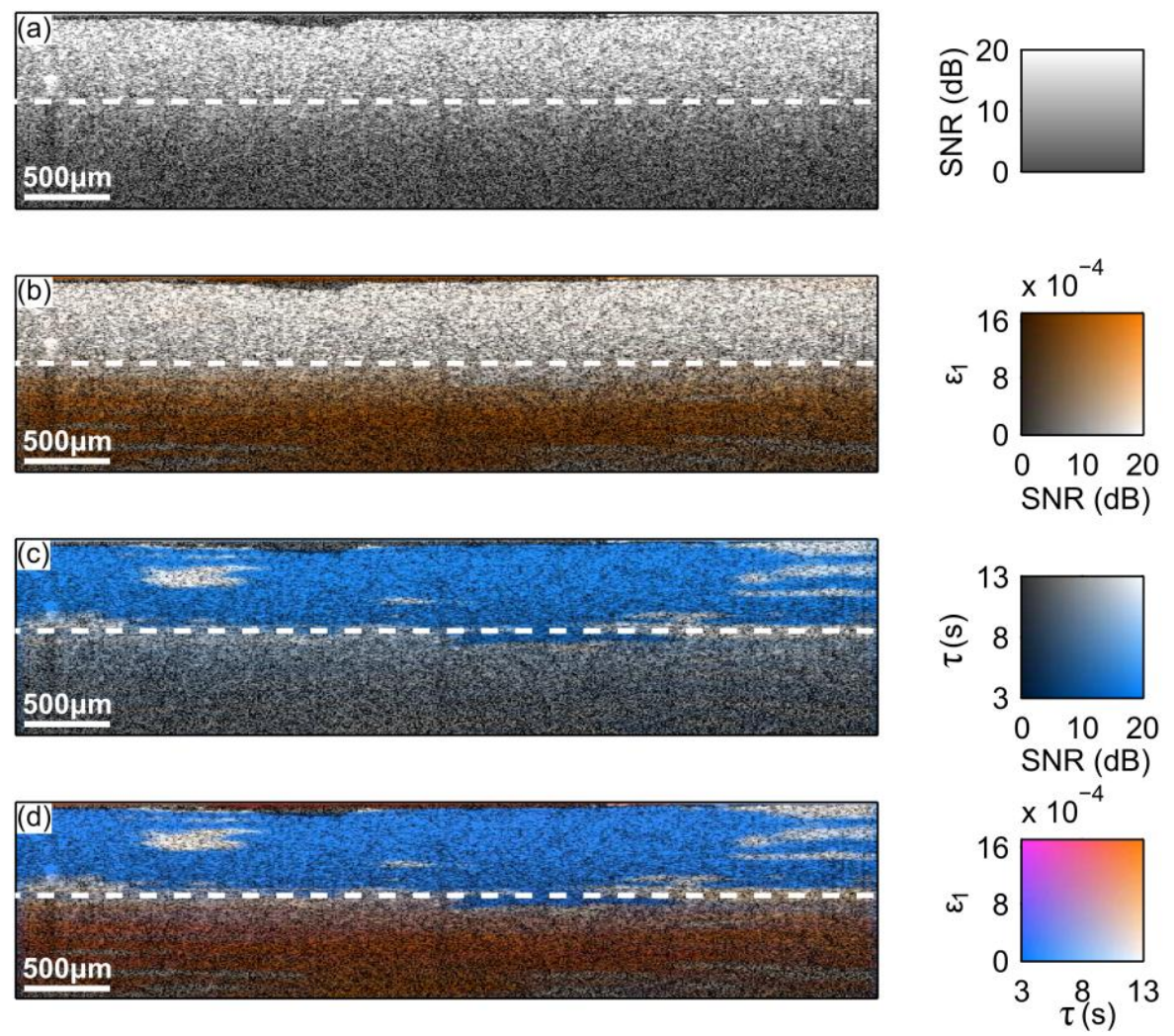

Figure 5. (a) OCT B-scan image of a dual-layer silicone phantom, overlaid with estimated (b) viscoelastic strain, $\varepsilon_{1}$; (c) viscoelastic time constant, $\tau$; and (d) dual-parameter $\left(\varepsilon_{1}, \tau\right)$ image. Layer boundary is indicated by the dashed white line. 


\subsection{Excised animal tissue}

Imaging of viscoelasticity using OCE was performed on a rat gastrocnemius muscle. An OCT B-scan image of the muscle is presented in figure 6(a). The imaged side of the muscle was partially sheathed by fascia (Moore et al. 2013), a collagenous layer that may be identified in OCT by its higher backscatter. Parameter maps were generated using a rectangular smoothing kernel with a $250-\mu \mathrm{m}$ lateral and $70-\mu \mathrm{m}$ axial window size. The dual-parameter viscoelastogram presented in figure $6(\mathrm{~b})$ shows distinct layers corresponding to the superficial connective tissue and adjacent muscle tissue, with connective tissue presenting higher $\varepsilon_{1}$ and lower $\tau$ values (orange) and the muscle tissue presenting intermittent regions of lower $\varepsilon_{1}$ and higher $\tau$ (blue), and lower $\varepsilon_{1}$ and lower $\tau$ (transparent). Muscle close to the surface on the right hand side of the image presents regions of higher $\varepsilon_{1}$ and higher $\tau$ (purple). At low OCT SNR, the displacement estimated using the phase-sensitive method, described in Section 2.3, is zero biased (Goodman 1985); therefore, the measured strain rate and the viscoelastic parameters are also zero biased in regions of low SNR. This accounts for much of the transparent, or colourless, regions in the viscoelastogram.
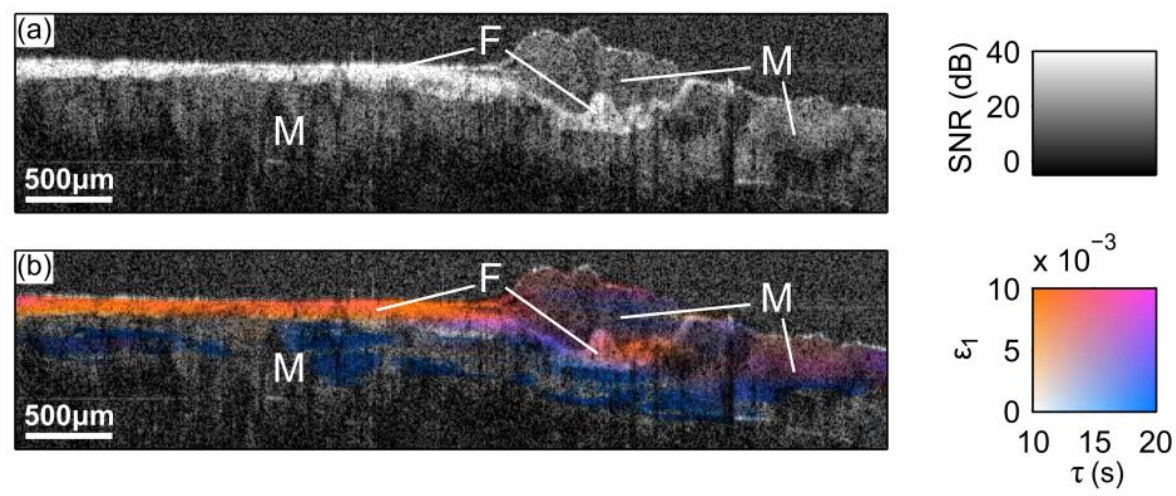

Figure 6. (a) OCT B-scan image of a rat gastrocnemius muscle, (b) overlaid with a dual-parameter $\left(\varepsilon_{1}\right.$ and $\tau$ ) image. F: fascia, and M: muscle tissue.

Imaging of viscoelasticity using OCE was also performed on a mouse abdominal muscle. An OCT B-scan image of the muscle is presented in figure 7(a), in which we can see a layered structure comprising: the higher scattering peritoneum (consisting primarily of collagenous connective tissue (Nagy and Jackman 1998)); the lower scattering extraperitoneal fatty tissue; and the inner layer of transverse abdominal muscle (O'Rahilly 1983). The dual-parameter viscoelastogram is presented in figure 7(b). Viscoelastic parameters were calculated using a rectangular smoothing kernel with a 125$\mu \mathrm{m}$ lateral and 35- $\mu \mathrm{m}$ axial window size. The peritoneal layer exhibits higher $\varepsilon_{1}$ and lower $\tau$ values, and the transverse muscle fibres exhibit lower $\varepsilon_{1}$ and higher $\tau$. The extraperitoneal fat layer is less defined due to the heterogeneity of tissue (Nagy and Jackman 1998), as well as due to lower scattering and, therefore, lower SNR. The fat layer presents generally higher $\varepsilon_{1}$ values with interspersed regions 
of high $\tau$ in its thicker parts. The delineation of layers in mouse abdomen is less pronounced than in the previous, structurally simpler case of rat muscle fascia.
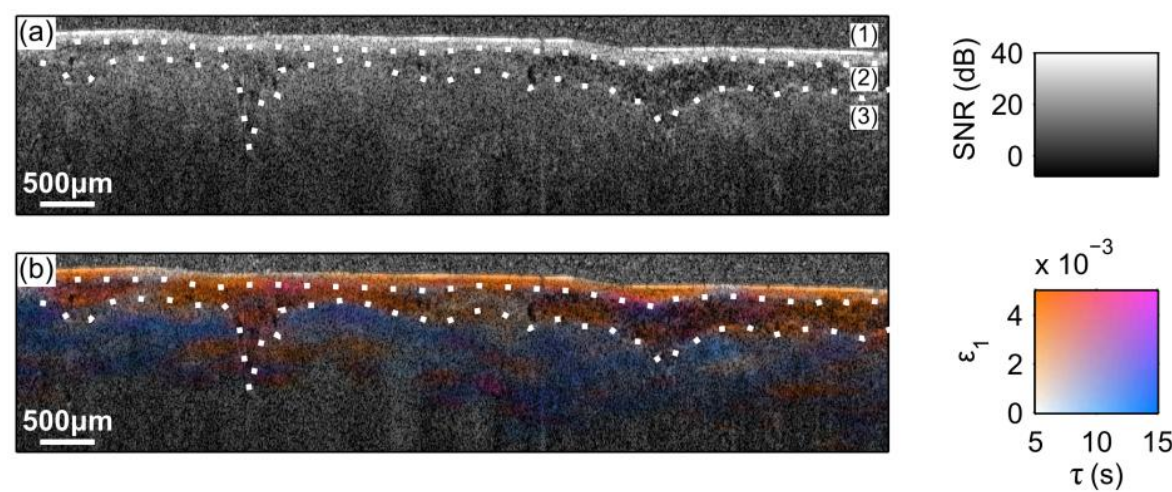

Figure 7. (a) OCT B-scan image of mouse abdominal muscle and surrounding tissue. (b) OCT overlaid with a dual-parameter ( $\varepsilon_{1}$ and $\tau$ ) image. Tissue layers are: (1) peritoneum; (2) extraperitoneal fat; and (3) inner layer of transverse abdominal muscle. Layer interfaces are marked by white dotted lines.

\section{Discussion}

Clinical application of imaging of soft tissue viscoelasticity using ultrasound and MRI has been investigated (Insana et al. 2004; Sinkus et al. 2005a; Qiu et al. 2008), and has demonstrated the ability to discriminate between benign and malignant breast lesions. In the work presented here, we have demonstrated the capacity to image viscoelasticity using an optical method, providing prospects for higher spatial resolution than ultrasound (Sridhar and Insana 2007; Urban et al. 2013) or MRI (Guo et al. 2013) methods. Further investigation is needed to ascertain whether imaging of viscoelasticity on the scale provided by optical imaging can provide useful additional information in biomechanics investigations and clinically relevant scenarios. The resolution of the OCE technique is variable and sample specific. Experimentally, we have found that spatial averaging was necessary to produce a sufficiently high signal-to-noise ratio in the measured strain rate. We have presented viscoelastograms estimated with a $50 \mu \mathrm{m}$ axial fitting range of displacement used in strain estimation (Kennedy et al. 2012), and with $250 \mu \mathrm{m}$ (lateral) by $70 \mu \mathrm{m}$ (axial) (boxcar) averaging in the dual-layer phantom and rat gastrocnemius muscle, and $125 \mu \mathrm{m}$ (lateral) by $35 \mu \mathrm{m}$ (axial) averaging in the mouse abdominal transection. The ideal lateral resolution of the system is determined by the lateral boxcar kernel size, and the ideal axial resolution, by the sum of the axial boxcar kernel size and the axial fitting length. However, the ideal resolution can only be reached given perfect step strain responses in the imaged samples. The effective resolution will likely be further degraded by the presence of boundary effects between mechanical features (Kennedy et al. 2014d). It may be possible to improve resolution by implementing Gaussian spatial filtering or by performing repetitive creep measurements on the same sample, adding 
another dimension for averaging. Furthermore, any improvement in strain sensitivity in compression phase-sensitive OCE is likely to allow for spatial averaging over a smaller kernel.

The interpretation of viscoelastic contrast provided by our technique requires the consideration of both, viscoelastic strain and viscoelastic time constant parameters. This is naturally facilitated by the dual-parameter representation on a single colour scale used in this paper. We have generated our colour map following the framework presented by Geissbuehler and Lasser (2013) and Baum et al. (2006). By overlaying the dual-parameter viscoelastogram on the OCT B-scan, we also provide structural context to the estimated viscoelasticity. Such dual-parameter images are common in fluorescence imaging, and overlays have been used in multi-modality imaging, such as MRI/PET (Baum et al. 2006). Singleparameter overlay images have also been previously explored in cardiovascular imaging using polarization-sensitive OCT (Villiger et al. 2013).

Previous work in compression OCE has been restricted to imaging the elastic (not viscoelastic) response of tissue, with care needed to avoid the time-dependent viscoelastic effects (Kennedy et al. 2009; Kennedy et al. 2014a). In the context of the Kelvin-Voigt model, such imaging probes the instantaneous elastic response, i.e., elastic strain, $\varepsilon_{0}$, which is independent of the viscoelastic strain and time constant (Tschoegl 1989). Therefore, viscoelastograms may provide new insight into tissue mechanics compared with conventional strain elastograms. However, as mentioned in Section 2.2, elastic strain and viscoelastic strain are not absolute measures: accompanying measurements of the local stresses are required to quantify mechanical moduli. In simple phantom geometries, local stress could be inferred from the force applied at the surface by the gravitational load; however, in tissue possessing mechanical heterogeneity and uneven boundaries, such estimation of local stress will only likely be accurate throughout the sample bulk with application of inverse methods (Kennedy et al. 2013). This is in contrast to the viscoelastic time constant, $\tau$, which is independent of the applied load and directly quantifiable. Thus, comparison of the measured time constant may be made between different measurement systems and samples. In figures 6(b) and 7(b), we observe measured $\tau$ values between 5 and $10 \mathrm{~s}$ in the collagenous connective tissue. The viscoelastic time constant of collagen fibrils was reported to be $7 \pm 2 \mathrm{~s}$ by Shen et al. (2011), which is within our measurement range. Fibrillar collagen is considered to be the dominant source of viscoelasticity in connective tissue (Burlew and Weber 2000).

In table 1, we can see that the precision in estimating viscoelastic parameters in the stiffer RT601 5:1:0 and in the softer P7676 4:1:0 is generally lower than for phantoms in the middle of the stiffness range. The dynamic range of our technique, i.e., the range of measureable viscoelastic parameters, is limited but can be shifted by varying acquisition and loading parameters. For instance, by increasing the B-scan acquisition speed, the maximum measurable strain rate (limited by phase wrapping) is increased. The disadvantage is that the increased acquisition speed raises the OCT noise floor. Increasing acquisition speed is likely to improve the precision for tissue exhibiting a lower viscoelastic time constant. This performance can be further improved by implementing a phase-unwrapping algorithm, similar to that demonstrated in compression OCE (Kennedy et al. 2014c). However, improvements at the high strain rates exhibited at the start of viscoelastic creep are likely to be limited by phase decorrelation, i.e., the decorrelation of the speckle patterns in consecutive B scans, as discussed in Chin 
et al. (2014). Further evaluation is required to access the accuracy of the measured viscoelastic parameters, for example, by comparing to existing mechanical testing systems. Furthermore, accuracy and precision should be tested for a wider range of viscoelasticities than reported here, which may be produced using fibrin or poly(vinyl alcohol) cryogels (PVA-C) (Lamouche et al. 2012) or gelatine (Insana et al. 2004).

Tissue often possesses uneven surface topography. Experimentally, we have observed that surface irregularities often correspond to heterogeneities beneath the sample surface in viscoelastograms. By placing the tissue on a compliant silicone layer, as described in Section 2.4, we have observed that these surface effects are minimized. As observed in previous work (Kennedy et al. 2014d), a compliant layer dissipates sharp variations in stress, and acts to provide a more uniform stress at the tissue-compliant layer interface. By minimizing surface-induced variations in stress, the use of the compliant layer likely results in the measured viscoelastic strain contrast becoming more linearly related to the true creep modulus. Further investigation is required to evaluate the effect of dimensions and mechanical properties of the layer on the stress distribution and uniformity, as well as its effect on the sensitivity of the measured viscoelastic parameters.

We have estimated only two of the four parameters in the Kelvin-Voigt model, viscoelastic strain and viscoelastic time constant. We briefly discuss the other two parameters, elastic strain and viscous strain. It is challenging to extract elastic strain from our model due to the high rate of deformation in the early viscoelastic response causing high rates of phase wrapping. In any case, measurement of elastic strain is readily performed by compression OCE (Kennedy et al. 2014c). The two methods could be performed sequentially by modifying the loading hardware to incorporate micro-scale actuation, as described in (Kennedy et al. 2009). Viscous strain, $\varepsilon_{2}$, is already estimated using our technique, but there are several issues to be considered. As mentioned in Section 2.2, viscous strain accounts for the steady-state loss of volume of a material, e.g., the exudation of PDMS oils in phantoms, and the exudation of fluids in tissue. To minimize the effects of friction at the sample boundaries in compression, we have applied lubricating fluid to sample surfaces: PDMS oil in phantoms and saline in tissue. We have observed experimentally that the measured viscous strain is dependent on the amount of lubrication used; therefore, the measurements of $\varepsilon_{2}$ were not representative of the true viscous behaviour. It may be possible to hold constant the contribution of the lubrication towards the measured $\varepsilon_{2}$ if the amount of lubricating fluid were to be controlled. Such an effect would likely not arise if this technique were to be applied to in vivo or in situ scenarios, as measured viscous strain should represent the viscous flow expected in the tissues' physiological environments. In these cases it could potentially be used to generate additional contrast.

Previous works in ultrasound (Insana et al. 2004; Sridhar and Insana 2007) and MRI (Sinkus et al. 2005a; Sinkus et al. 2005b) have implemented more complex models of viscoelastic creep behaviour: primarily, a dual-exponential model derived from two series Kelvin-Voigt units and characterized by two viscoelastic strains and time constants. For our technique, a single-exponential model proved sufficient to generate contrast in phantoms and muscle tissue. If the dynamic range or the sensitivity of strain rate measurements were to be improved, a more complex model of viscoelastic creep might 
provide additional information, but further investigation would be required to analyse the trade-off in the increase in number of parameters with the goodness of fit in implementing higher order models (Akaike 1974). Furthermore, the Kelvin-Voigt model is one-dimensional and does not account for the anisotropy of tissue, which is present in muscle. Contrast in the muscle viscoelastograms will likely vary based on the direction of the muscle fibres with respect to the direction of compression. Further investigation is required to access the feasibility of OCE in measuring anisotropic viscoelasticity as it will likely require not only a different viscoelastic model but also the measurement of viscoelasticity in different sample orientations (Van Loocke et al. 2009) or, alternatively, the reconstruction of the full strain tensor in three dimensions (Sinkus et al. 2005a).

Our technique has the potential to be extended to three-dimensional imaging. A limit in threedimensional acquisition is the trade-off between acquisition rate and the density of sampling over the field of view, as the acquisition rate of co-located OCT scans affects the dynamic range of our technique. As described in Section 2.3, we have acquired B-scans at a rate of $10 \mathrm{~Hz}$, formed from $1000 \mathrm{~A}$-scans taken with a $100 \mu$ s period. Using the same OCT system, recent work in compression OCE (Kennedy et al. 2014b) has demonstrated the ability to acquire A-scans with a $10 \mu$ s period, forming threedimensional OCE volumes in $5 \mathrm{~s}$. Therefore, it may be possible to sample viscoelastic creep at a density of 10,000 A-scans per C-scan (for example, $100 \mathrm{~A}$-scans in a B-scan and $100 \mathrm{~B}$-scans in a C-scan), whilst maintaining the same co-located acquisition rate of $10 \mathrm{~Hz}$. A faster OCT system, with megahertz A-scan rates, has been presented by Wieser et al. (2010), although the phase sensitivity of the system was not reported; therefore, further investigation is required to assess the feasibility of such a system for phase-sensitive measurements.

\section{Conclusion}

In this paper, we have demonstrated the ability of OCE to measure the viscoelastic creep response by extracting the parameters $\varepsilon_{1}$ and $\tau$, which have been used to form B-scan dual-parameter images. We have demonstrated viscoelastic creep imaging, showing repeatable measurements of viscoelastic parameters in tissue-simulating phantoms and have demonstrated the ability to generate additional contrast to that of OCT imaging. We have observed viscoelastic contrast in excised rat and mouse muscle tissue, discriminating between tissue structures on the sub-millimetre scale. Viscoelasticity imaging using OCE may provide opportunities to improve the sub-millimetre to micro-scale mechanical characterization of tissues and disease pathologies, as well as provide contrast complementary to existing OCE techniques.

\section{Acknowledgements}

We would like to thank Kelsey Kennedy and Lixin Chin for providing guidance and assistance in phantom fabrication and data processing. Philip Wijesinghe acknowledges the support of the William and Marlene Schrader Scholarship provided by The University of Western Australia. This project is supported with funding from the National Health and Medical Research Council, Australia; Cancer Council WA; and the Australian Research Council. 


\section{References}

Akaike H 1974 A new look at the statistical model identification IEEE Transactions on Automatic Control $196716-723$

Alonso-Caneiro D, Karnowski K, Kaluzny BJ, Kowalczyk A and Wojtkowski M 2011 Assessment of corneal dynamics with high-speed swept source optical coherence tomography combined with an air puff system Optics Express 1915 14188-14199

Baum KG, Helguera M and Krol A 2006 Genetic algorithm automated generation of multivariate color tables for visualization of multimodal medical data sets in Color and Imaging Conference Society for Imaging Science and Technology pp. 138-143.

Burlew BS and Weber KT 2000 Connective tissue and the heart: Functional significance and regulatory mechanisms Cardiology Clinics 183 435-442

Chan RC, Chau AH, Karl WC, Nadkarni S, Khalil AS, Iftimia N, Shishkov M, Tearney GJ, Kaazempur-Mofrad MR and Bouma BE 2004 OCT-based arterial elastography: Robust estimation exploiting tissue biomechanics Optics Express 1219 4558-4572

Chang J, Moon W, Cho N, Yi A, Koo H, Han W, Noh D-Y, Moon H-G and Kim S 2011 Clinical application of shear wave elastography (SWE) in the diagnosis of benign and malignant breast diseases Breast Cancer Research and Treatment 1291 89-97

Chau AH, Chan RC, Shishkov M, MacNeill B, Iftimiia N, Tearney GJ, Kamm RD, Bouma BE and Kaazempur-Mofrad MR 2004 Mechanical analysis of atherosclerotic plaques based on optical coherence tomography Annals of Biomedical Engineering 3211 1494-1503

Chin L, Curatolo A, Kennedy BF, Doyle BJ, Munro PRT, McLaughlin RA and Sampson DD 2014 Analysis of image formation in optical coherence elastography using a multiphysics approach Biomedical Optics Express 59 2913-2930

Crecea V, Ahmad A and Boppart SA 2013 Magnetomotive optical coherence elastography for microrheology of biological tissues Journal of Biomedical Optics 1812121504

de Bruin DM, Bremmer RH, Kodach VM, de Kinkelder R, van Marle J, van Leeuwen TG and Faber DJ 2010 Optical phantoms of varying geometry based on thin building blocks with controlled optical properties Journal of Biomedical Optics 152 025001-025001-10

Discher D, Dong C, Fredberg J, Guilak F, Ingber D, Janmey P, Kamm R, Schmid-Schönbein G and Weinbaum S 2009 Biomechanics: Cell research and applications for the next decade Annals of Biomedical Engineering 375 847-859

Ford MR, Dupps WJ, Rollins AM, Roy AS and Hu ZL 2011 Method for optical coherence elastography of the cornea Journal of Biomedical Optics 161

Friedl P and Alexander S 2011 Cancer invasion and the microenvironment: Plasticity and reciprocity Cell 1475 992-1009

Geissbuehler M and Lasser T 2013 How to display data by color schemes compatible with red-green color perception deficiencies Optics express $2189862-9874$

Geyer MJ, Brienza DM, Chib V and Wang J 2004 Quantifying fibrosis in venous disease: Mechanical properties of lipodermatosclerotic and healthy tissue Advances in Skin \& Wound Care 17 131-142

Goodman JW 1985 Statistical Optics (New York: Wiley-Interscience).

Guo J, Hirsch S, Fehlner A, Papazoglou S, Scheel M, Braun J and Sack I 2013 Towards an elastographic atlas of brain anatomy PLOS ONE 88 e 71807

Ingber D 2003 Mechanobiology and diseases of mechanotransduction Annals of Medicine $358564-$ 577 
Insana MF, Pellot-Barakat C, Sridhar M and Lindfors KK 2004 Viscoelastic imaging of breast tumor microenvironment with ultrasound J Mammary Gland Biol Neoplasia 94 393-404

Kennedy BF, Curatolo A, Hillman TR, Saunders CM and Sampson DD 2011 Speckle reduction in optical coherence tomography images using tissue viscoelasticity Journal of Biomedical Optics $\mathbf{1 6}$ 2020506

Kennedy BF, Hillman TR, McLaughlin RA, Quirk BC and Sampson DD 2009 In vivo dynamic optical coherence elastography using a ring actuator Optics Express 1724 21762-72

Kennedy BF, Kennedy KM and Sampson DD 2014a A review of optical coherence elastography: Fundamentals, techniques and prospects IEEE Journal of Selected Topics in Quantum Electronics 202 272-288

Kennedy BF, Koh SH, McLaughlin RA, Kennedy KM, Munro PR and Sampson DD 2012 Strain estimation in phase-sensitive optical coherence elastography Biomedical Optics Express 38 186579

Kennedy BF, Malheiro F, Chin L and Sampson DD 2014b Three-dimensional optical coherence elastography by phase-sensitive comparison of C-scans Journal of Biomedical Optics, (Preprint)

Kennedy BF, McLaughlin RA, Kennedy KM, Chin L, Curatolo A, Tien A, Latham B, Saunders CM and Sampson DD 2014c Optical coherence micro-elastography: Mechanical-contrast imaging of tissue microstructure Biomedical Optics Express 57 2113-2124

Kennedy KM, Es'haghian S, Chin L, McLaughlin RA, Sampson DD and Kennedy BF 2014d Optical palpation: optical coherence tomography-based tactile imaging using a compliant sensor Optics Letters 3910 3014-3017

Kennedy KM, Ford C, Kennedy BF, Bush MB and Sampson DD 2013 Analysis of mechanical contrast in optical coherence elastography Journal of Biomedical Optics 1812 121508-121508

Lamouche G, Kennedy BF, Kennedy KM, Bisaillon CE, Curatolo A, Campbell G, Pazos V and Sampson DD 2012 Review of tissue simulating phantoms with controllable optical, mechanical and structural properties for use in optical coherence tomography Biomedical Optics Express 36 13811398

Lee GYH and Lim CT 2007 Biomechanics approaches to studying human diseases Trends in Biotechnology 253 111-118

Liang X, Oldenburg AL, Crecea V, Chaney EJ and Boppart SA 2008 Optical micro-scale mapping of dynamic biomechanical tissue properties Optics Express 1615 11052-11065

Mierke CT 2012 Endothelial cell's biomechanical properties are regulated by invasive cancer cells Molecular BioSystems 86 1639-1649

Moore KL, Dalley AF and Agur AM 2013 Clinically Oriented Anatomy (Lippincott Williams \& Wilkins).

Nagy JA and Jackman RW 1998 Anatomy and physiology of the peritoneal membrane Seminars in Dialysis $11149-56$

Nahas A, Bauer M, Roux S and Boccara AC 2013 3D static elastography at the micrometer scale using Full Field OCT Biomedical optics express 410 2138-2149

O'Rahilly R 1983 Basic Human Anatomy: A Regional Study of Human Structure (Philadelphia: Saunders).

Oldenburg AL and Boppart SA 2010 Resonant acoustic spectroscopy of soft tissues using embedded magnetomotive nanotransducers and optical coherence tomography Physics in Medicine and Biology 5541189

Papandrinopoulou D, Tzouda V and Tsoukalas G 2012 Lung compliance and chronic obstructive pulmonary disease Pulmonary Medicine 2012542769 
Parker KJ, Doyley MM and Rubens DJ 2011 Imaging the elastic properties of tissue: The 20 year perspective Physics in Medicine and Biology $561 \mathrm{R} 1$

Qi W, Chen R, Chou L, Liu G, Zhang J, Zhou Q and Chen Z 2012 Phase-resolved acoustic radiation force optical coherence elastography Journal of Biomedical Optics 1711110505

Qin Z, Buehler MJ and Kreplak L 2010 A multi-scale approach to understand the mechanobiology of intermediate filaments Journal of Biomechanics $\mathbf{4 3} 1$ 15-22

Qiu Y, Sridhar M, Tsou JK, Lindfors KK and Insana MF 2008 Ultrasonic viscoelasticity imaging of nonpalpable breast tumors: Preliminary results Academic Radiology 1512 1526-1533

Sandrin L, et al. 2003 Transient elastography: A new noninvasive method for assessment of hepatic fibrosis Ultrasound in Medicine and Biology 2912 1705-1713

Shen Zhilei L, Kahn H, Ballarini R and Eppell Steven J 2011 Viscoelastic properties of isolated collagen fibrils Biophysical Journal 10012 3008-3015

Sinkus R, Tanter M, Catheline S, Lorenzen J, Kuhl C, Sondermann E and Fink M 2005a Imaging anisotropic and viscous properties of breast tissue by magnetic resonance-elastography Magnetic Resonance in Medicine $\mathbf{5 3} 2372-87$

Sinkus R, Tanter M, Xydeas T, Catheline S, Bercoff J and Fink M 2005b Viscoelastic shear properties of in vivo breast lesions measured by MR elastography Magnetic Resonance in Medicine 232 15965

Song S, Huang Z and Wang RK 2013 Tracking mechanical wave propagation within tissue using phase-sensitive optical coherence tomography: Motion artifact and its compensation Journal of Biomedical Optics 1812121505

Sridhar M and Insana MF 2007 Ultrasonic measurements of breast viscoelasticity Medical Physics 34 $124757-67$

Srivastava A, Verma Y, Rao KD and Gupta PK 2011 Determination of elastic properties of resected human breast tissue samples using optical coherence tomographic elastography Strain 47 175-87

Tschoegl NW 1989 The Phenomenological Theory of Linear Viscoelastic Behavior: An Introduction (Springer-Verlag).

Urban MW, Pislaru C, Nenadic IZ, Kinnick RR and Greenleaf JF 2013 Measurement of viscoelastic properties of in vivo swine myocardium using lamb wave dispersion ultrasound vibrometry (LDUV) IEEE Trans. Med. Imag 322 247-261

Van Loocke M, Simms CK and Lyons CG 2009 Viscoelastic properties of passive skeletal muscle in compression - Cyclic behaviour Journal of Biomechanics 428 1038-1048

Villiger M, Zhang EZ, Nadkarni SK, Oh W-Y, Vakoc BJ and Bouma BE 2013 Spectral binning for mitigation of polarization mode dispersion artifacts in catheter-based optical frequency domain imaging Optics Express 2114 16353-16369

Wang RK, Kirkpatrick S and Hinds M 2007 Phase-sensitive optical coherence elastography for mapping tissue microstrains in real time Applied Physics Letters 9016164105

Wang S and Larin KV 2014 Shear wave imaging optical coherence tomography (SWI-OCT) for ocular tissue biomechanics Optics Letters 39 1 41-44

Wang Y and Insana MF 2013 Viscoelastic properties of rodent mammary tumors using ultrasonic shear-wave imaging Ultrasonic Imaging 352 126-45

Wieser W, Biedermann BR, Klein T, Eigenwillig CM and Huber R 2010 Multi-megahertz OCT: High quality 3D imaging at 20 million A-scans and 4.5 GVoxels per second Optics Express 1814 1468514704 
Yang Y, Bagnaninchi PO, Ahearne M, Wang RK and Liu K-K 2007 A novel optical coherence tomography-based micro-indentation technique for mechanical characterization of hydrogels Journal of The Royal Society Interface 417 1169-1173

Yoon G, Kab Kim Y, Eom K and Na S 2013 Relationship between disease-specific structures of amyloid fibrils and their mechanical properties Applied Physics Letters 1021011914 